\title{
PARALLEL FAULT SYSTEMS WITH EVOLVING SELF-SIMILAR SLIDING ZONES
}

\author{
A.V. Dyskin \\ School of Civil and Resource Engineering \\ The University of Western Australia \\ 35 Stirling Hwy, Crawley, WA 6009, Australia \\ Fax: (61-8) 64881044 \\ E-mail: adyskin@cyllene.uwa.eduau
}

Submitted: 20 March 2007

Accepted: 9 July 2007

\begin{abstract}
Catastrophic fault sliding is preceded by the development of sliding zones which grow further driven by the excess of the shear stress over friction at the loci of initiation. This growth is strongly affected by the interaction between the sliding zones. We propose a model of development of such zones based on two major simplifications. Firstly, each sliding zone is modelled as a disc-like shear crack driven by a pair of concentrated forces representing the excess of the shear stress over friction at the loci of initiation. Secondly, the interaction between these cracks is modelled based on the assumption that the distribution of their sizes is self-similar and the self-similarity is maintained in the process of their growth. We show that for parallel cracks the latter is only possible if the sliding zones are localised in a narrow layer. In this case the exponent and the prefactor
\end{abstract}


of the distribution function are uniquely determined. The addition of new sliding zones does not change the distribution but rather increases the upper cut-off. This happens either by instantaneous growth of each added sliding zone to the maximum size producing the strongest microseismic event or by initiating a cascade of intermediate growth producing a series of smaller events. We determine the energy distribution associated with the cascade and the probability of hazardous events. We show that measuring the statistical properties of seismic energy alone is not sufficient for determining the parameters of the model; monitoring of fault deformation is also needed.

Keywords: Shear Crack, Crack Interaction, Self-similar Distributions, Effective Characteristics, Stress Intensity Factors

\section{Introduction}

One of the main mechanisms of earthquakes is catastrophic sliding over a fault, which occurs rapidly and then propagates dynamically (e.g., Scholz, 1990). In principle, if the original stress state consisting of the gravitational and tectonic components is known together with the stress redistribution caused by the Earth's crust structure and properties, then complete determination of the magnitude of the resulting seismic event can be achieved provided that the strength parameters of the fault are known. The most difficult is the determination of the fault strength properties, since the parameters determined by retrospective analysis of the post failure situation (post-mortem examination) do not have to be relevant to a next sliding which will happen in conditions changed by the previous fault sliding. Also a possibility of some strength 
increase with time (healing, e.g., Scholz, 1990; Abinante and Knopoff, 1995) should be taken into account. In this case the main tool for prediction of the sliding or, at least the risk assessment, is seismic monitoring. The major challenge in seismic monitoring is the development of interpretation methods which, in order to be realistic, have to be based on the understanding the mechanics of catastrophic fault sliding.

In many cases the mechanical behaviour of the fault is controlled by the rock mass structure encompassing a number of scales. Then the assumption of self-similarity in distributions of microstructural elements becomes a major simplifying factor in an otherwise usually intractable problem. There is a strong evidence of self-similar properties of both rocks and the Earth’s crust (e.g., Sadovskiy, 1983; Scholz and Aviles, 1986; Scholz, 1990; Redner, 1990; Olding, 1992; Barton and Zoback, 1992; Turcotte, 1993, 2004; Gillespie et al., 1993; Yamamoto et al., 1993; Dubois, 1998).

The appearance of self-similar structures is usually attributed to the critical state of the material (e.g., Bak and Tang, 1989; Chopard and Droz, 1998), however, the particular mechanism of formation of self-similar distributions, particularly distributions of cracks and fractures, is poorly understood. The most popular approach is to consider the fractures as clusters of connected defects (e.g., Sahimi and Goddard, 1986; Nishiuma et al., 1996; Chakrabati and Benguigui, 1997; Mishnaevsky, 1998) which, near the critical state (i.e. at the percolation threshold), have self-similar distributions. It should however be noted that only in the $2 \mathrm{D}$ picture these structures actually break the material. In the real 3D world the formation of such structures does not affect the connectedness of the body. 
Dyskin (2001, 2002) proposed a mechanism of developing isotropic self-similar distributions of disc-like cracks, based on crack interaction and leading to a self-similar distribution of crack sizes, with the distribution function proportional to the inverse fourth power of the crack radius. Essential in this model is the stable growth of the cracks, which is provided by a special type of loading, viz by a couple of concentrated forces applied at the centre of every disc-like crack. Dyskin (2005) proposed a 2D model of growth of sliding zones and demonstrated that the self-similar distributions of sizes of sliding zones can maintain themselves only in the case of isotropically oriented faults and in the case of sliding zones localised in a narrow zone. The 2D model may however have only restricted applications because the 3D effects can play a significant role in fracture propagation, especially in the presence of compression (e.g., Dyskin et al., 2003).

In this paper we develop a 3D model of interaction of shear cracks in self-similar systems and the evolution of sliding zones. The paper is organised as follows. In Section 2 we introduce a model of evolution of a single sliding zone in a pre-existing fault. We assume that the sliding zone is developed due to a local deterioration of friction in the fault independently of the deformation rate or slip velocity. This locus is then considered as a region of excess of shear force and serves as a driving force to propagate the sliding zone further. We model such a sliding sone as a planar disc-like shear crack in homogeneous elastic medium, ignoring the real discontinuities as the purpose of this study is to investigate a mechanism of emergence of self-similar distributions. We assume that the features that introduce lengths scales, such as fault 
curvature or blocks are very large as compared to the dimensions of the sliding zones. We use a conventional Linear Elastic Fracture Mechanics criterion of their propagation (see, for instance, Scholz, 1990), the fracture energy being a macroscopic substitute for microscopic mechanisms of sliding over the fault. Section 3 treats the effect of interaction between these sliding zones. It is based on the assumption that because the sliding zones could not be born equal the interaction only exaggerates the size inequality and produces a wide distribution of sizes (sliding zones of similar sizes are in low concentration and hence do not interact directly; the interaction is essential only between the zones of considerably different sizes). Under this assumption a model case of chaotically oriented disc-like shear cracks is considered and demonstrated that interaction leads the emergence of a self-similar crack distribution. To circumvent the technical difficulties pertinent to the realistic case of parallel cracks, a self-similar size distribution needs to be considered and checked whether it survives the process of evolution of the sliding zones. The necessary formalism is introduced in Section 4. Based on this, Section 5 demonstrates that in the case of distributed parallel sliding zones the self-similar distribution could not be formed. Section 6 considers another limiting case of coplanar sliding zones and demonstrates the stability of their selfsimilar size distribution. Section 7 infers the statistical parameters of seismic energy distribution associated with the evolution of self-similar distributions of sliding zones.

\section{A model of sliding zone}

We consider a fault in a meta-stable state arising when sufficient time has elapsed after a previous sliding such that a cementation or another healing process has restored the cohesion. This condition will be expressed in terms of the large-scale (macroscopic with 
respect to the dimensions of the sliding zones) shear, $\tau$, and normal, $\sigma_{N}$, stress components, acting on the fault plane by assuming that it is the cohesion that makes the fault stable, while if the cohesion is removed, the fault is in moving equilibrium. This is expressed as follows (compressive stresses are assumed positive)

$\tau<c+\sigma_{N} \tan \varphi, \quad \tau=\sigma_{N} \tan \varphi$

where $\varphi$ is the friction angle and $c$ is the current cohesion.

The stress variations with time and the time effects, like cyclic loading caused by tidal stress and neighbouring seismic events or delayed fracture producing friction deterioration can generate local sliding zones at weak places of the fault, which, as yet, do not affect the fault stability. The local sliding zones can also be formed if the fault is locally hit by a tensile wave from a neighbouring seismic event with a magnitude exceeding the normal compressive stress (this mechanism was considered by Dyskin et al., 1998). We will call the sliding zones initiated by one of these mechanisms the initial sliding zones. Such a zone is shown in Figure 1 where the fault is sketched with its middle section, AA'. The initial sliding zone (denoted by $\mathrm{CC}^{\prime}$ in Figure 1) is characterised by the sliding resistance, $\tau_{I}$, which is considerably lower than the largescale friction on the fault:

$\tau_{I}=c_{I}+\sigma_{N} \tan \varphi_{I}<\sigma_{N} \tan \varphi$

The initial sliding zone will propagate to a sliding zone of a certain size (denoted by BB' in Figure 1). We assume that the cementation in this zone is broken and the resistance to 
sliding is only provided by dilation on asperities in accordance to a conventional model (Patton, 1966).

We firstly consider the case when the sliding zone is alone (the following sections will consider the interaction of self-similarly distributed sliding zones). Then its development can be modelled by a shear crack loaded at a central part (segment CC' in the middle section) by shear tractions $\tau-\tau_{I}$, Figure 2a. Since we are going to consider potentially extensive development of the sliding zones to sizes much greater than the size of the initial zone we will, as a further simplification, model the sliding zone as a crack sheared by a pair of concentrated forces of average magnitude $F=S\left(\tau-\tau_{I}\right)$, where $S$ is the area of the initial sliding zone, Figure $2 b$. 


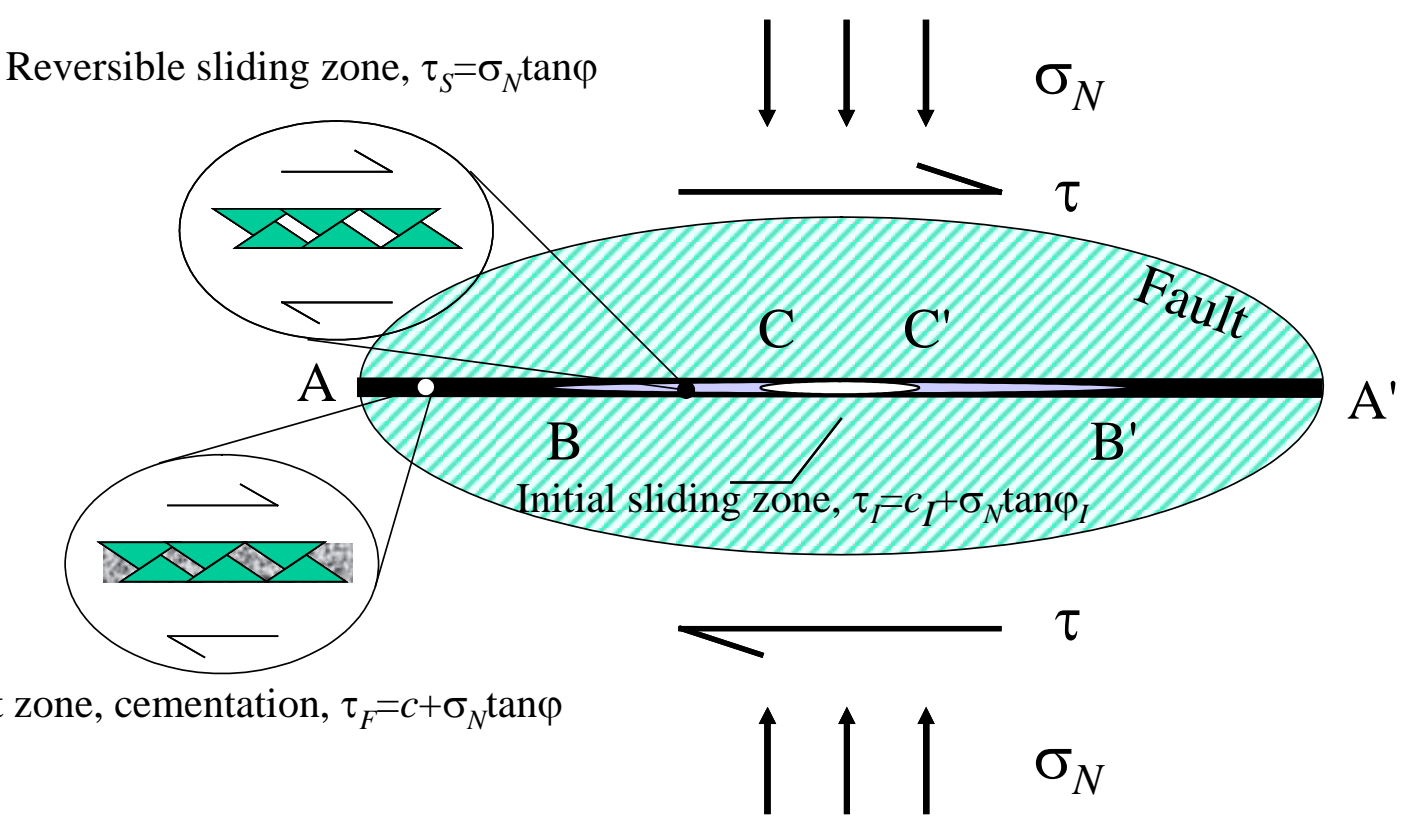

Fig. 1. Schematic representation of a fault with a sliding zone. A cross section is shown. The sliding zone with cross-sectional dimensions BB' is developed from an initial sliding zone CC'. It is assumed that the large-scale friction in the sliding zone is provided by interacting asperities with negligible friction between the asperity surfaces, such that the sliding over this part of the fault could, in the first approximation be assumed reversible. 


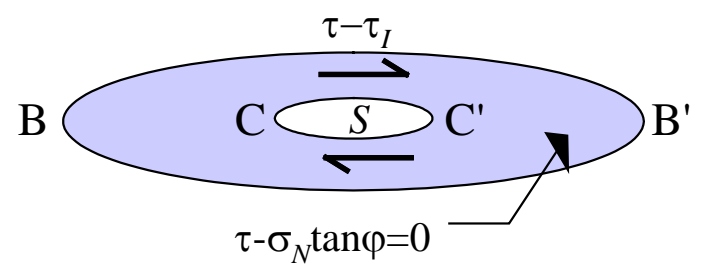

(a)

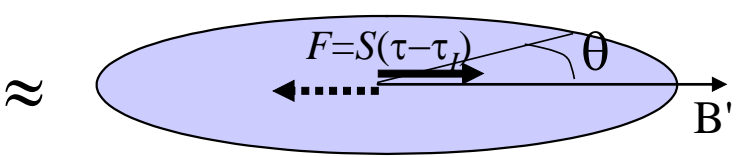

(b)

Fig. 2. A model of the sliding zone: (a) a representation of the sliding zone as a shear crack loaded at the central part (marked in white) of area $S$ which corresponds to the initial sliding zone; (b) modelling the sliding zone as a shear crack loaded by a pair of concentrated forces of magnitude $F=S\left(\tau-\tau_{I}\right)$, the angle $\theta$ is a polar angle

The growth of such sliding zones is controlled by the fault microstructure, in particular the fault roughness and the strength of the gouge. Macroscopically, these factors will be represented by the macroscopic (with respect to the characteristic size of the fault asperities) fracture energy, $\Gamma$. Then, neglecting the contribution of the dilatant opening of the fault to the energy release rate a crack propagation type criterion can be formulated for the growth of a sliding zone:

$\frac{1-v^{2}}{E} K_{I I}^{2}+\frac{1+v}{E} K_{I I I}^{2}=\Gamma$

where $E, v$ are Young's modulus and Poisson's ratio of the surrounding material of the Earth's crust which, in this simplified model, is assumed isotropic and homogeneous elastic. 
For a disc-like crack shown in Figure 2b, the Mode II and III stress intensity factors $K_{I I}$ and $K_{I I I}$ have at point B' the following form (e.g., Tada et al, 1985)

$K_{I I}=\frac{F}{(\pi R)^{3 / 2}} \frac{2}{2-v}(1+v) \cos \theta, \quad K_{I I I}=-\frac{F}{(\pi R)^{3 / 2}} \frac{2}{2-v}(1-2 v) \sin \theta$

where $R$ is the crack radius, the polar angle $\theta$ is shown in Figure 2 .

Substitution of equations (4) into (3) shows that the propagation criterion for the sliding zone is not uniform over the crack contour, which means that even if the initial shape was circular it will not be maintained in the process of the growth of the sliding zone. Nevertheless, since the present analysis is mainly concerned with the effects of interaction, as an initial approximation we consider a simplified model of the sliding zone. In this model the sliding zone will still be treated as a disc-like shear crack with the propagation criterion based on a certain average value of the stress intensity factors over the crack contour. Subsequently, since the expressions for both $K_{I I}$ and $K_{I I I}$ have the same factor $F(\pi R)^{-3 / 2}$, the criterion of growth of the sliding zone can, in this approximation, be expressed as

$\frac{F}{(\pi R)^{3 / 2}}=K_{*}$,

where $K_{*}$ is a force representation of the average fracture energy $\bar{\Gamma}$.

$\frac{1-v^{2}}{E} K_{*}^{2}=\bar{\Gamma}$ 
We assume that $K_{*}$ is scale-independent (with respect to the scales exceeding the characteristic dimensions of the fault asperities).

As evident from (5), the sliding zone grows in a stable manner with the radius increasing with the force as $F^{2 / 3}$. This implies that the growth of sliding zones is controllable and hence excludes the dynamic phase. It means that the growth of separate sliding zones by themselves cannot cause catastrophic sliding. The mechanism of large seismic events should therefore be sought in the effect of interaction between the sliding zones. This mechanism is considered in the following section.

\section{Interacting shear cracks. Emergence of self-similarity}

Interaction between sliding zones literally means that each zone propagates under the action of a superposition of the external stress and stress disturbances, generally nonuniform, generated by all other sliding zones. When the number of sliding zones is large the modelling of propagation of interacting sliding zones becomes quite complicated.

A considerable simplification can be achieved by employing the notion of selfsimilarity in the distribution of the sliding zones, which is suggested by the GuttenbergRichter law universally observed in the records of both natural and technogenic seismic events (e.g., Scholz, 1990; Gibowicz and Kijko, 1994). Self-similarity implies that the scaling is described by power law, which is the major simplifying factor in addressing the problem of interaction as will be shown in the following section. More importantly, in some cases, including the case of sliding zones considered, the interaction itself can 
produce self-similar distributions as will be demonstrated below (the consideration below will make use of the method developed by Dyskin, 2001, 2002 for Mode I cracks).

We consider a material with cracks growing in a stable manner, for instance driven by pairs of equivalent concentrated forces applied to the centres of the crack faces. Suppose that the cracks are distributed randomly. Then, even if all cracks were initially of the same size and were loaded by exactly the same forces, the interaction will make them grow differently such that a certain size distribution of cracks will emerge. One can then assume that the difference in the crack sizes will only increase with their growth. Thus the interaction of such cracks can be modelled in the asymptotics of large distribution of sizes (Salganik, 1973) assuming that: (i) cracks of close sizes do not interact directly and; (ii) the interacting cracks are very different in size. Then each crack can be considered in an equivalent medium with effective characteristics determined by all cracks of smaller sizes.

We start the consideration with a somewhat artificial case of isotropic distribution of sliding zones (shear cracks), meaning that the faults subject to sliding are randomly oriented. We will further assume that the asperity-resisted sliding can be reversible such that small deviations of the shear stresses from the friction stress can cause both increase and reduction in the crack sliding. This assumption is essential for the proposed use of the effective characteristic theory since the basis of it - the consideration of a crack in an effective medium determined by smaller cracks - presumes that the effective medium is elastic at least incrementally at the stress near the equilibrium point, such 
that loading and unloading follow the same path. Modelling of this case will provide the concept which will then be extended to the case of parallel faults which is more realistic for describing fault sliding.

Direct modelling of the growth of large numbers of interacting cracks is quite complex, especially in 3D. A significant simplification can be achieved if the crack growth, more precisely its stable phase, is formulated in terms of the average stress intensity factors $\left\langle K_{I}\right\rangle,\left\langle K_{I I}\right\rangle,\left\langle K_{I I I}\right\rangle$, where the averaging is presumed over all possible mutual locations and, possibly, orientations of the interacting cracks ${ }^{1}$.

We now consider each crack as being in an effective medium whose characteristics determined by smaller cracks, the calculation of the average stress intensity factors involves two scales. The effective medium determines the overall displacement discontinuities over the crack surface, therefore the computations involving the effective medium correspond to the scale macroscopic with respect to the small cracks. The stress intensity factors on the other hand reflect the stress concentration in a vicinity of the crack contour at the distances closer than the dimensions of the small cracks. Hence the stress intensity factors represent the stress distributions at the scale microscopic with respect to the small cracks. Consequently, the average stress intensity factors should be determined from the distribution of the displacement discontinuity close to the crack

\footnotetext{
${ }^{1}$ The usual perception is that the fracture processes are described by extreme quantities, be it a maximum stress or minimum strength. This is of course relevant to the stage when a critical crack is formed and dynamically propagates producing the ultimate failure. However if the ultimate failure is preceded by a considerable stage of multiple crack accumulation/growth without dynamics, the use of average stress intensity factors would more adequate.
} 
contour. Since here the scale of consideration is microscopic with respect to the small cracks, the respective stress concentration corresponds to the part of the original material free from the small cracks. Hence, the elastic moduli relating this part of displacement discontinuity and the stress intensity factors are at this stage the elastic moduli of the material. For the isotropic case the average stress concentrations can therefore be expressed in the following form (see Dyskin, 2002 for details)

$$
\frac{\left\langle K_{I}\right\rangle}{K_{I}^{0}}, \frac{\left\langle K_{I I}\right\rangle}{K_{I I}{ }^{0}}, \frac{\left\langle K_{I I I}\right\rangle}{K_{I I I}{ }^{0}} \sim \frac{E_{0}}{E}
$$

where $E$ is the effective Young's modulus, $E_{0}$, is the Young's modulus of the material, $K_{I}{ }^{0}, K_{I I}{ }^{0}, K_{I I I}{ }^{0}$ are the stress intensity factors which would have characterised the crack had there be no interaction with small cracks. Then the criterion of propagation of the sliding zone (5) assumes the form

$$
K_{*}=\frac{F}{(\pi R)^{3 / 2}} \frac{E_{0}}{E} .
$$

Suppose that at a certain value of the load, $F$, the cracks have the distribution function $f(R), R_{0} \leq R \leq R_{\max }$. Here $R_{0}$ is the radius which the crack (sliding zone) would have without the interaction, $R_{\max }$ is the maximum radius attained under the given value $F$. It will also be assumed that this crack distribution can approximately be considered as the wide distribution of sizes.

Based on this assumption, the effective moduli for the material with cracks up to the given size can be calculated. Consider cracks with radii between $R$ and $R+\mathrm{d} R$. Because the number of these cracks is low, they shall be considered as non-interacting and 
placed in an effective medium determined by cracks of radii smaller than $R$. Let the Young's modulus and Poisson's ratio of this medium be $E(R)$ and $v(R)$. Then, following Salganik's (1973) differential self-consistent method, the effective characteristics for the medium with the new cracks of radii between $R$ and $R+\mathrm{d} R$ are obtained as the effective characteristics of a medium with moduli $E(R)$ and $v(R)$. The concentration of these new cracks is:

$d v(R)=N R^{3} f(R) d R$

where $N$ is the number of cracks per unit volume.

The concentration of these new cracks being infinitesimal allows neglecting the interaction between them. For the case of pure shear disc-like cracks (no opening or closure, i.e. normal displacement is continuous through the crack) the effective moduli in the approximation of low concentrations $(v<<1)$ have the form (see Appendix):

$$
E=E_{0}\left[1-\frac{64}{45} \frac{1-v_{0}^{2}}{2-v_{0}} v\right], \quad v=v_{0}+\frac{32}{45} \frac{1-v_{0}^{2}}{2-v_{0}}\left(1-2 v_{0}\right) \nu
$$

Equations (9) can be further simplified by taking into account that since $0 \leq v_{0} \leq 0.5$,

$0.5 \leq \frac{1-v_{0}^{2}}{2-v_{0}} \leq 0.536$

such that with the accuracy of $7.2 \%$ this fraction can be replaced with $1 / 2$. Subsequently, 
$E \approx E_{0}\left[1-\frac{32}{45} v\right], \quad v \approx v_{0}+\frac{16}{45}\left(1-2 v_{0}\right) v$

According to the differential self-consistent method, at each step when we apply formulae (9) or the approximation (11), the moduli obtained at the previous step play the role of $E_{0}$ and $v_{0}$. Consequently,

$$
\begin{aligned}
& E(v+d v)=E(v)\left[1-\frac{32}{45} d v\right] \\
& v(v+d v)=v(v)+\frac{16}{45}[1-2 v(v)] d v
\end{aligned}
$$

We now obtain from the first equation of the above system and equation (8) that

$$
\frac{d E}{d R}=-\frac{32}{45} E N R^{3} f(R)
$$

Expressing $E$ from (7) differentiating it with respect to $R$ and substituting into (12) one obtains

$$
f(R)=\frac{135}{64 N} \frac{1}{R^{4}}
$$

The distribution function should satisfy the usual normalisation condition

$$
\int_{R_{0}}^{R_{\max }} f(R) d R=1
$$

This gives 
$\frac{1}{R_{0}^{3}}-\frac{1}{R_{\max }{ }^{3}}=\frac{64}{45} N$

Expressing $R_{0}$ - the crack radius in the absence of interaction - from (5):

$R_{0}=\frac{1}{\pi}\left(\frac{K_{*}}{F}\right)^{2 / 3}$

and substituting into equation (15) one has

$\frac{R_{\max }}{R_{0}}=\left[1-\frac{64 F^{2}}{45 \pi^{3} K_{*}^{2}} N\right]^{-1 / 3}$

As the expression in the square brackets tends to zero, the ratio $R_{\max } / R_{0} \rightarrow \infty$ which can be interpreted as complete (catastrophic) sliding of the fault. The corresponding force magnitude is denoted as $F_{\text {max }}$ :

$F_{\max }=\frac{3 \pi K_{*}}{8} \sqrt{\frac{5 \pi}{N}}$

From here one obtains

$$
\frac{R_{\max }}{R_{0}}=\left[1-\frac{F^{2}}{F_{\max }^{2}}\right]^{-1 / 3}
$$

The power law (13) together with $R_{\max } / R_{0} \rightarrow \infty$ can be interpreted as an emergence of a self-similar distribution in the crack arrangement considered. In more complex cases it is technically more difficult to trace the emergence of self-similar distributions. What we propose instead is to determine whether the self-similar distributions are stable with 
respect to the described type of crack growth. This, necessary condition of the selfsimilar distributions, will be analysed in the following sections.

\section{Mechanics of materials with self-similar crack distributions}

\subsection{General considerations}

Let the crack distribution be self-similar such that there is no characteristic size in the microstructure. According to Dyskin (2004) a material with such crack distribution should be modelled simultaneously at all scales by a continuous set of continua (the $H$ continua) with the volume element sizes, $H$, assuming all values. In this case, all continuum quantities should be also functions of scale, $H$. Then all characteristics of the continua become the power functions of $H$. Furthermore, it was proven that all tensorial properties should scale isotropically, i.e. all tensorial components should scale with the same exponent. In particular, the tensors of elastic moduli, $\mathbf{C}$, and compliances, $\mathbf{A}$, in a Cartesian co-ordinate frame $\left(x_{1}, x_{2}, x_{3}\right)$ must scale for any crack orientations and any material anisotropy as

$$
\begin{aligned}
& C_{i j k l}(H)=c_{i j k l} H^{\alpha}, \quad A_{i j k l}(H)=a_{i j k l} H^{\beta} \\
& i, j, k, l=1,2,3, \quad \alpha=-\beta
\end{aligned}
$$

for all non-zero components of the prefactors. Here $\alpha$ and $\beta$ are the common scaling exponents for the tensors of elastic moduli and compliances respectively.

The prefactors and exponents can be determined from the following system of equations, if the expressions for the scaling for the contribution of cracks to the 
compliances or moduli, $\Delta A_{i j k l}=\Delta a_{i j k l} H^{\rho_{A}}$ or $\Delta C_{i j k l}=\Delta c_{i j k l} H^{\rho_{C}}$ are known for the case of non-interacting cracks. Then using the differential self-consistent method and taking into account that according to the dimension analysis the exponent $\rho_{A}=\beta-1$ (or $\rho_{C}=\alpha-1$ ) one obtains the following system of scaling equations (see details in Dyskin, 2004)

$\beta a_{i j k l}=\Delta a_{i j k l} \quad$ or $\quad \alpha c_{i j k l}=\Delta c_{i j k l}$

Consider, for instance, the first system (The second system can be treated similarly). This is generally a system of 21 equations for 22 unknowns, $a_{i j k l}$ and $\beta$. Since the prefactors for both compliances and the increments have the same units, one of the compliance prefactors can be chosen arbitrarily, while the other prefactors and the exponent can be found by solving this system.

For a special case of self similar crack distributions $f(R)=w R^{-4}$, to which distribution (13) belongs (with the concentration factor $w=135 / 64 N$ ), it is shown by Dyskin (2002) that the assumptions of the wide distribution of sizes are satisfied and that the differential self-consistent method can be used to determine $\Delta c_{i j k l}$.

Furthermore, in line with (6), the average SIFs scale as

$$
\left\langle K_{I}\right\rangle,\left\langle K_{I I}\right\rangle,\left\langle K_{I I I}\right\rangle \sim H^{-\alpha}
$$

We consider now two important particular cases. 


\subsection{Isotropic self-similar distributions of disc-like shear cracks}

In the case of randomly oriented pure shear cracks the second system of scaling equations (20) can be obtained from (11) by considering only the crack contribution to the moduli and then by formal replacement of $v$ with $w$. We also take into account that the Poisson's ratio being bounded has the scaling exponent equal to zero, i.e. $v=$ const . This produces the following system of scaling equations:

$$
\left\{\begin{array}{l}
\alpha E=-E \frac{32}{45} w \\
0=\frac{16}{45}(1-2 v) w
\end{array}\right.
$$

The solution of system (22) reads

$$
E=e H^{\alpha}, \quad \alpha=-\frac{32}{45} w, \quad v=0.5
$$

where $e$ is a normalising prefactor. It is seen that both Young's modulus and Poisson's ratio scale according to power laws. The exponent for the Young's modulus is proportional to the concentration factor $w$. It is interesting that the value for the Poisson's ratio is found to be 0.5 , suggesting that materials with self-similar distributions of isotropically oriented pure shear cracks are incompressible. This is because self-similar distributions are formally unbound. Hence, formally, for finite w, the total crack concentration is infinite such that the properties of materials with selfsimilar crack distributions are fully controlled by the crack behaviour. In this case the incompressibility is a result of the absence of any normal relative displacements of the faces of shear cracks. 


\subsection{Parallel disc-like shear cracks}

Consider now a case of parallel disc-like shear cracks which could model sliding zones over a distributed set of parallel faults. Suppose the cracks are oriented perpendicular to the $x_{3}$ axis and distributed self-similarly with the distribution function $f(R)=w R^{-4}$. For this case the effective compliances can be found from the general solution by Vavakin and Salganik (1978) for a transverse-isotropic material with disc-like cracks parallel to the plane of isotropy. The Hooke's law for a transverse isotropic material is expressed in the co-ordinate set $\left(x_{1}, x_{2}, x_{3}\right)$ through the compliances as follows

$$
\begin{aligned}
& \varepsilon_{11}=A_{11} \sigma_{11}+A_{12} \sigma_{22}+A_{13} \sigma_{33} \\
& \varepsilon_{22}=A_{12} \sigma_{11}+A_{11} \sigma_{22}+A_{13} \sigma_{33} \\
& \varepsilon_{33}=A_{13} \sigma_{11}+A_{13} \sigma_{22}+A_{33} \sigma_{33} \\
& \varepsilon_{23}=\frac{1}{2} A_{44} \sigma_{23} \\
& \varepsilon_{13}=\frac{1}{2} A_{44} \sigma_{13} \\
& \varepsilon_{12}=\left(A_{11}-A_{22}\right) \sigma_{12}
\end{aligned}
$$

Vavakin and Salganik's (1978) solution, after the contribution of the crack in the normal strain in the direction perpendicular to the cracks (the $x_{3}$ axis) is set to zero assumes the form:

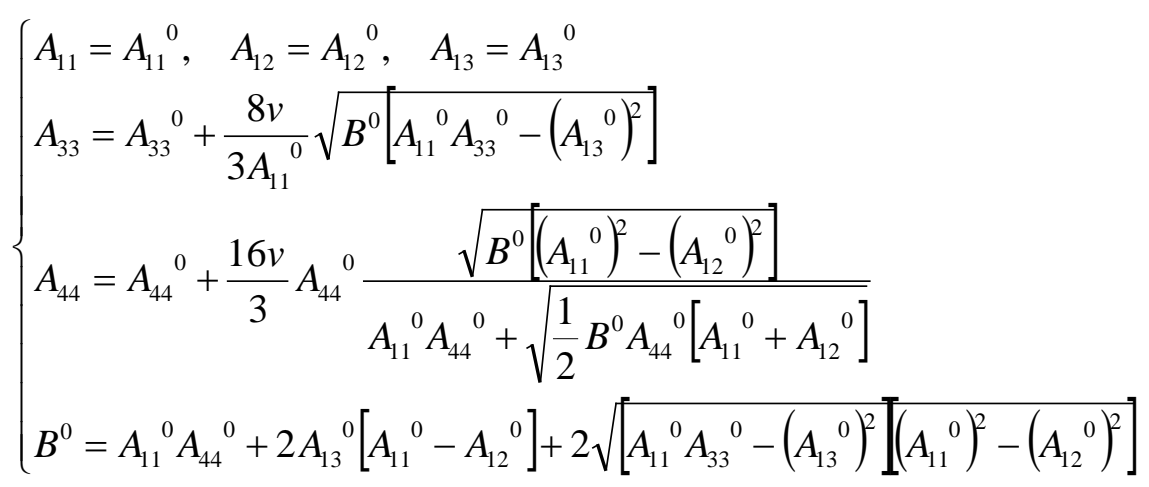


Here $A_{11}{ }^{0}, A_{12}{ }^{0}, A_{13}{ }^{0}, A_{33}{ }^{0}, A_{44}{ }^{0}$ are the compliances of the material.

The scaling equations (the first system in (20)) can be obtained by replacing $v$ with $w$ bringing $A_{i i}{ }^{0}$ to the left-hand sides and then replacing $A_{i i}-A_{i i}{ }^{0}$ with $\beta a_{i i}$ and, finally, replacing $A_{i i}{ }^{0}$ with $a_{i i}$ in the remaining parts. This yields the following scaling equations

$$
\left\{\begin{array}{l}
\beta a_{11}=0, \quad \beta a_{12}=0, \quad \beta a_{13}=0 \\
\beta a_{33}=\frac{8 w}{3 a_{11}} \sqrt{B\left[a_{11} a_{33}-\left(a_{13}\right)^{2}\right]} \\
\beta=\frac{16 w}{3} \frac{\sqrt{B\left[\left(a_{11}\right)^{2}-\left(a_{12}\right)^{2}\right]}}{a_{11} a_{44}+\sqrt{\frac{1}{2} B a_{44}\left[a_{11}+a_{12}\right]}} \\
B=a_{11} a_{44}+2 a_{13}\left[a_{11}-a_{12}\right]+2 \sqrt{\left.\left[a_{11} a_{33}-\left(a_{13}\right)^{2}\right]\left(a_{11}\right)^{2}-\left(a_{12}\right)^{2}\right]}
\end{array}\right.
$$

Suppose $\beta \neq 0$. Then the first three equations of (26) yield $a_{11}=a_{12}=a_{13}=0$. Then the third equation produces $\beta=0$. This contradiction means that the scaling exponent vanishes. As a result we obtain the following scaling law:

$$
A_{i j}=a_{i j} H^{\beta}, \quad \beta=0
$$

\section{Stability of self-similarity in the case of distributed sets of growing shear cracks}

We are now in a position to check whether the above self-similar distributions of shear cracks are stable with respect to the mechanism of crack growth described in Section 2. Assuming that each homogenisation scale $H \sim R$ (in the $H$-continuum only cracks of sizes $R>H$ can be seen) and substituting scaling (21) into the expression for the stress 
intensity factors (4), (6) and then the result into the criterion of shear crack growth (5) one obtains

$K_{*} \sim R^{-\alpha-3 / 2}$.

From here, since $K_{*}$, is assumed to be scale independent, we obtain an equation for the scaling exponent $-\alpha-3 / 2=0$. Therefore, if the cracks are to grow keeping the selfsimilarity the exponent of the moduli scaling cannot be arbitrary, but should satisfy

$\alpha=-3 / 2$

For isotropic distributions of disc-like shear cracks this, according to the scaling law (23) corresponds to

$w=135 / 64$

Thus the concentration factor of the crack distribution remains constant. This means that in order to maintain the concentration factor constant the crack growth must only affect the lower and upper cutoffs of the distribution (both $R_{\max }$ and $R_{0}$ get increased in accordance with the normalisation equations (14)).

In the case of parallel shear cracks, because the scaling of non-vanishing components of compliances or moduli is characterised by the same exponent, the scaling of the average stress intensity factors can be still obtained from the equations (6). Then the considerations of the previous section can be repeated and the condition of stability (29) can be obtained. However, for the case of a single set of parallel cracks this condition cannot be satisfied due to the trivial scaling $(\alpha=\beta=0)$. Therefore, for such crack 
distributions, the self-similarity cannot be maintained: it will be destroyed by crack growth. Consequently, one cannot expect that the crack growth will be accompanied by self-similar seismic emission which contradicts the Guttenberg-Richter law. This suggests that the case of distributed cracks locations is not realistic and a situation of cracks localised in a narrow band should be considered. This will be accomplished in the following section.

\section{Stability of self-similar distributions in the case of localised sets of parallel sliding zones}

The instability of a self-similar distribution of parallel cracks with respect to the crack growth came from the fact that the scaling exponent vanishes. This, in its own turn, is a consequence of the fact that these cracks do not contribute to some components of compliances that characterise the transverse-isotropic $H$-continua which model the material with a single set of distributed parallel cracks. Therefore, in order to find a stable arrangement of parallel cracks, one needs to find a situation when the cracks influence all essential components of compliances. An obvious candidate for this is a localised distribution of parallel cracks, i.e. the distribution in which all cracks are concentrated within a thin layer, Figure 3. (In order to maintain the self-similarity the layer should be infinitesimally thin; in reality its thickness should be much smaller than the lower cutoff of the crack radii, $R_{0}$.) We will model such a set of coplanar cracks as a Winkler layer with the shear stiffness $k$ defined as $\tau=k \Delta u_{s}$, where $\Delta u_{s}$ is the shear displacement discontinuity over the layer (difference in displacements at opposite boundaries of the layer) in response to shear load $\tau$. 


\section{Cross-section of fault}

Fig. 3. Coplanar sliding zones in a fault: a cross sectional view

Under the assumption of self-similar crack distribution, the stiffnesses should scale with the same exponent (since they are components of a diagonal tensor relating the stress vector and displacement discontinuity vector):

$k \sim H^{\alpha}$

Suppose the cracks are distributed according to the following distribution function

$$
f(R)=\frac{\lambda}{R^{m}}
$$

Consider an equivalent 2D $\mathrm{H}$-continuum which models the Winkler layer. This $\mathrm{H}$ Winkler layer comprises all cracks of the size up to $R \sim H$. Transition to the scale $H+d H$ leads to the addition of new cracks occupying relative area of the fault $d w=\pi \lambda R^{2-m} d R$. These new cracks increase the average (effective) stress, $\tau_{\text {eff, }}$ by the factor of $(1-d w)^{-1}$, because the presence of cracks simply reduce the intact area subjected to loading with a given total force. This results in the reduction of effective stiffness by the factor of $(1-d w)$. Subsequently 


$$
\frac{d k}{k}=-\pi \lambda R^{2-m} d R d k / k=-\pi \lambda R^{2-m} d R
$$

Obviously, the power law is only possible if $m=3$ :

$$
f(R)=\frac{\lambda}{R^{3}}
$$

Solution of the differential equation (33) gives

$$
\alpha=-\pi \lambda, \quad k \sim R^{-\pi \lambda}, \quad \tau_{e f f} \sim R^{\pi \lambda}
$$

The influence of the interaction on the average stress intensity factors scales inversely to the scaling of stiffness, therefore the criterion of crack grows reads

$$
K_{*} \sim \frac{F}{(\pi R)^{3 / 2}} R^{\pi \lambda} \sim R^{\pi \lambda-3 / 2}
$$

The self-similarity will be preserved if

$$
\lambda=\frac{3}{2 \pi}
$$

The total dimensionless concentration of cracks with sizes in the range from $R_{0}$ to $R_{\max }$ is

$$
\Omega=\int_{R_{0}}^{R_{\max }} R^{2} f(R) d R=\frac{3}{2 \pi} \ln \frac{R_{\max }}{R_{0}}
$$

Thus, the self similar distribution of parallel sliding zones in an infinitesimally thin layer is stable with respect to their growth. 
This result can obviously be applied to a system of parallel faults, as long as the interaction between the sliding zones belonging to different faults can be neglected. This can for instance happen when sliding occurs in different parts of the faults such that the distance between the sliding zones belonging to different faults is greater than the upper cut-off of the size distribution of the sliding zones.

\section{Self-similar cascades and the resulting distribution of seismic energy}

If we assume that the self-similarity of the size distribution of sliding zones is maintained, then the process of their evolution can be described as follows. The new sliding zones which are initially the zones of average radius $R_{0}$ induced in the fault will violate self-similarity. Therefore, the self-similarity will have to be restored by growing these new cracks (or some of them) to the highest sizes (radii) or by propagating them to some intermediate sizes followed by increasing the sizes of some other cracks. This cascade of propagating sliding zones will result in a new distribution with the same minimal radius $R_{0}$ but with an increased maximal one, $R_{\max }$.

In order to model this cascade of propagating cracks consider a level $R$. Since the cracks grow in such a way that the concentration factor, $\lambda$, remains constant (see (37)), the number of crack grown to radius $R$ should equal the number of cracks of radius $R$ starting their growth in the process of rearrangement. Let $g(R)$ be a fraction of cracks grown to radius $R$. Due to self-similarity this distribution should be expressed by a power law with a certain exponent $k$ : 
$g(R)=\frac{\gamma}{R^{k}}$

Subsequently the distribution of cracks grown to radius $R$ is (see also summary in Table 1)

$$
f(R) g(R)=\frac{\lambda \gamma}{R^{3+k}}
$$

Consider now the energy distribution of seismic events emitted by the cascade. Suppose the seismic event is associated with a growth of a crack (sliding zone) from a certain size $R_{1}$ to size $R$. We will assume that this growth happens dynamically such that the energy emitted by the crack $\mathrm{E}(R)$ is equal to the change in elastic energy associated with the displacement of the crack faces. Since the crack growth is caused by the crack interaction, represented in this model by some uniform effective stress $\tau_{\text {eff }}$, the energy is $\mathrm{E}(R) \sim \tau_{\text {eff }}\left(R^{3}-R_{1}^{3}\right)$. Assuming that the majority of growing cracks considerably increase their sizes, $R>>R_{1}$ and taking into account the last equation of (35) and equation (37) one obtains

$$
\mathrm{E}(R)=\kappa R^{9 / 2}
$$

One can now express the energy distribution by the probability of events with energy greater that a certain level $U$ :

$$
P\{\mathrm{E}>U\}=P\left\{R>\left(\frac{U}{\kappa}\right)^{\frac{2}{9}}\right\}=\int_{\left(\frac{U}{\kappa}\right)^{\frac{2}{9}}}^{\infty} \frac{\pi \lambda \gamma}{R^{3+k}} d R
$$


This results in

$P\{\mathrm{E}>U\}=\frac{\pi \lambda \gamma}{k+2}\left(\frac{\kappa}{U}\right)^{\frac{2}{9}(k+2)}$

This is a Guttenberg-Richter law expressed in terms of the energy rather than magnitude. After relating the exponent $2(k+2) / 9$ to the observed one it is possible to find $k$.

\section{Determination of the model parameters. Risk assessment}

In order to determine the parameters of the model we consider statistical moments of the seismic energy:

$$
\left\langle\mathrm{E}^{n}\right\rangle=\int_{R_{0}}^{R_{\max }} \mathrm{E}(R)^{n} f(R) g(R) d R=\frac{\pi \lambda \gamma \kappa^{n}}{2+k-4.5 n}\left[\frac{1}{R_{0}^{2+k-4.5 n}}-\frac{1}{R_{\max }^{2+k-4.5 n}}\right]
$$

Thus by measuring the consecutive energy moments $\left\langle E^{n}\right\rangle$ one can determine remaining parameters, $\kappa, R_{0}, R_{\max }$ and the combination $\lambda \gamma$. One of the parameters in this combination, $\lambda$ or $\gamma$, should be determined independently. One possibility would be to evaluate $\lambda$ by monitoring the fault deformation, since this parameter together with $R_{0}$ and $R_{\max }$ determines the effective stiffness of the fault.

If these parameters were determined the earthquake hazard assessment could be performed in the cases when the mechanism of seismicity is dominated by the self- 
similar development of distribution of sliding zones. We note that the maximum energy of a seismic event has the form:

$\mathrm{E}_{\max }=\kappa R_{\max }^{9 / 2}$

If this energy exceeds the safe level, $\mathrm{E}_{\text {safe }}$, then the seismic event should be considered as hazardous. The risk (probability) of a hazardous seismic event can be estimated as

$$
P_{\text {hazard }}=\int_{\left(\mathrm{E}_{\text {safe }} / \kappa\right)^{2 / 9}}^{R_{\max }} g(R) d R=\left\{\begin{array}{l}
\frac{\gamma}{k-1}\left[\left(\frac{\kappa}{\mathrm{E}_{\text {safe }}}\right)^{\frac{2}{9}(k-1)}-\left(\frac{\kappa}{\mathrm{E}_{\max }}\right)^{\frac{2}{9}(k-1)}\right], \quad \text { if } k \neq 1 \\
\frac{2}{9} \ln \frac{\mathrm{E}_{\text {max }}}{\mathrm{E}_{\text {safe }}}, \quad \text { if } k=1
\end{array}\right.
$$

\section{Conclusion}

We have considered a specific mechanism of seismicity associated with the propagation of sliding zones within a fault in such a way that the distribution of sizes of the sliding zones maintains self-similar. This is of course a very strong assumption. If the persistent observations of Guttenberg-Richter law constitute a sufficient reason to believe that the self-similarity is a prevailing characteristic of the fault sliding, then we have a method of hazard assessment of earthquakes. It is essential that not all parameters needed for the hazard assessment could be determined from the microseismic measurements; these must be complemented by independent observations of the process of the fault deformation. 
Acknowledgment. The author acknowledges the financial support through the Australian Research Council Discovery Grant (DP0559737) and the financial support by the Australian Computational Earth Systems Simulator - Major National Research Facility (ACcESS MNRF). The author also wishes to express thanks to Elena Pasternak for valuable comments.

\section{References}

Abinante, M.S. and Knopoff, L. (1995), Quasidynamic model for earthquake simulations, Physical Rev. E, 52, No. 5, 5675-5678.

Bak, P. and Tang, C. (1989), Earthquakes as a self-organised critical phenomenon, J. Geophys. Res., 94B (11), 15,635-15,637.

Barton, C.A. and Zoback, M.D. (1992), Self-similar distribution and properties of macroscopic fractures at depth in crystalline rock in the Cajon Pass scientific drill hole. J. Geophys. Res., 97B (4), 5181-5200.

Chakrabati, B.K. and Benguigui, L.G., Statistical Physics of Fracture and Breakdown in Disordered Systems (Clarendon Press, Oxford 1997).

Chopard, B. and Droz, M., Cellular Automata Modelling of Physical Systems (Cambridge University Press 1998).

Dubois, J. Non-Linear Dynamics in Geophysics (John Wiley and Sons, Chichester, New York, Weinheim, Brisbane, Singapore, Toronto 1998).

Dyskin, A.V. (2001), Self-similar crack distributions: Mechanics of formation and method of modelling. Intern. J. Fracture, 112, L41-L46.

Dyskin, A.V. (2002), Self-similar crack patterns induced by spatial stress fluctuations. Fatigue and Fracture of Engineering Materials and Structures, 25, 187-200. 
Dyskin, A.V. (2004), Effective characteristics and stress concentrations in materials with self-similar microstructure. Int. J. Solids Struct., 42 (2) 477-502.

Dyskin, A.V., Mining-induced seismicity associated with self-similar propagation of sliding zones, In Controlling Seismic Risk. Proc. Sixth International conference on Rockburst and Seismicity in Mines (RaSiM6) (ed. Potvin, Y and Hudyma, M.), (UWA 2005) pp. 319-325.

Dyskin, A.V., Galybin A.N. and Brady, B.H., Catastrophic sliding over a fault caused by accumulation of dilation zones, In Mechanics of Jointed and Faulted Rock (MJFR-3), (ed. Rossmanith H.P.), (Balkema 1998), pp. 69-74.

Dyskin, A.V., Jewell, R.J., Joer, H., Sahouryeh, E. and Ustinov, K.B. (2003), Influence of shape and locations of initial 3-D cracks on their growth in uniaxial compression. Engg. Fracture Mechanics, 70, Issue 15, 2115-2136.

Gibowicz, S.J. and Kijko A., An introduction to Mining Seismology. (Academic Press, San Diego, New York, Boston, London, Sydney, Tokyo, Toronto 1994).

Gillespie, P.A., Howard, C.B., Walsh, J.J. and Watterson, J. (1993), Measurement and characterisation of spatial distribution of fractures. Tectonophysics, 226, 113-141.

Mishnaevsky, L., Damage and Fracture in Heterogeneous Materials. Modelling and Application to the Improvement of Drilling Tool. (Balkema, Rotterdam, Brookfield 1998).

Nishiuma, S., Hasegawa, Y. and Miyazima, S. (1996), Percolated cracks in a solid containing many initial defects. Fractals, 4 (3), 377-384.

Olding, N.E. (1992), Network properties of a two-dimensional nature fracture pattern, Pure and Applied Geoph., 138, 95-114. 
Patton, F.D. Multiple modes of shear failure in rock, In Proc. of the First Congress of ISRM (Lisbone 1966), 1, 509-513.

Redner, S. Fragmentation, In: Statistical Models for the Fracture of Disordered Media (ed. Herrmann, H.J., Roux, S.) (North-Holland, Amsterdam, Oxford, New York, Tokyo 1990), 321-348.

Sadovskiy, M.A., (1983), Distribution of preferential sizes in solids, Transactions USSR Academy of Sciences. Earth Science Series, 269 (1), 8-11.

Sahimi, M. and Goddard, J.D. (1986), Elastic percolation models for cohesive mechanical failure in heterogeneous systems, Physical Review B, 33 (11), 78487851.

Salganik, R.L. (1973), Mechanics of bodies with many cracks, Mech. of Solids, 8, 135143.

Scholz, C.H., The Mechanics of Earthquakes and Faulting. (Cambridge University Press, Cambridge, New York, Port Chester, Melbourne, Sydney 1990).

Scholz, C.H. and Aviles, C. The fractal geometry of faults and folding, In Earthquake Source Mechanics. Monograph series, 37. (ed. Das, S., Boatwright, J., Scholz, C.H.) (Washington, D.C.: American Geophysical Union 1986), 147-155.

Tada, H., Paris, P.C. and Irwin, G.R. The stress analysis of cracks. Handbook. Third edition. (New York: ASME Press(1985), Vol. II.

Turcotte, D.L. Fractals and Chaos in Geology and Geophysics. (Cambridge University Press 1993).

Turcotte, D.L. (2004), The relationship of fractals in geophysics to "the new science", Chaos, Solitons and Fractals, 19, 255-258. 
Vavakin, A.S. and Salganik, R.L. (1978), Effective elastic characteristics of bodies with isolated cracks, cavities, and rigid nonhomogeneities, Mech. of Solids, 13 (2), 8797.

Yamamoto, H., Kojima, K. and Tosaka, H., Fractal clustering of rock fractures and its modelling using cascade process, In Scale Effects in Rock Masses 93. (ed. Pinto da Cunha) (Balkema, Rotterdam 1993), 81-86.

\section{Appendix. Effective characteristics of an isotropic material with randomly oriented disc-like shear cracks in dilute concentrations}

Consider an isotropic material with chaotically distributed disc-like cracks and assume that the concentration of the cracks is low such that the interaction between the cracks can be neglected. Introduce a global Cartesian co-ordinate set $\left(x_{1}, x_{2}, x_{3}\right)$ and consider a volume element macroscopic with respect to the crack sizes. Suppose the volume element is loaded by uniform tractions which, in the absence of the cracks, would produce uniform stress field $\sigma_{i j}$ within the volume element.

Let $V_{i}$ be an $i^{\text {th }}$ component of displacement discontinuity through the crack integrated over the crack surface (this quantity has the units of volume). In the case of elastic material vector $V_{i}$ is linearly related to the applied stress which is considered to be uniform by the virtue of the uniform tractions applied to the volume element and the supposed absence of the crack interaction

$V_{k}=J_{k l m} \sigma_{l m}$ 
where $J_{k l m}$ is a tensor which depends on parameters of the crack and its orientation.

In accordance with the general theory, e.g., Salganik (1973) the effective compliances of the cracked body have the form

$$
S_{i k l m}=S_{i k l m}^{0}+\frac{1}{2} \int\left[n_{i} J_{k l m}+n_{k} J_{i l m}\right] F(\mathbf{Y}) d \mathbf{Y}
$$

Here $S^{0}{ }_{i k l m}$ is the tensor of compliances of the material, $n_{i}$ is the unit vector normal to the crack surface, $\mathbf{Y}$ is the vector of parameters of the shape and orientation of the crack. Summation over repeated indexes is presumed. The distribution function $F(\mathbf{Y})$ satisfies the following normalisation condition:

$$
\int F(\mathbf{Y}) d \mathbf{Y}=N
$$

where $N$ is the number of crack per unit volume.

Consider a crack and introduce a Cartesian co-ordinate set $\left(x^{\prime}{ }_{1}, x^{\prime}{ }_{2}, x^{\prime}{ }_{3}\right)$ with the $x^{\prime}{ }_{3}$ axis normal to the crack surface. For a disc like crack of radius $R$ we have in this co-ordinate set (Salganik, 1973)

$$
V_{k}^{\prime}=\frac{8 \pi}{3} \frac{1-v_{0}^{2}}{E_{0}} R^{3} \sigma_{k 3}^{\prime}
$$

where $\sigma_{i j}^{\prime}$ is the applied stress in this co-ordinate set, $E_{0}$ and $v_{0}$ are the Young's modulus and Poisson's ratio of the material. 
For shear cracks with $V_{3}=0$, tensor $J_{k l m}$ can be written in the co-ordinate set related to the crack as

$$
J_{k l m}^{\prime}=\frac{4 \pi}{3} \frac{1-v_{0}^{2}}{E_{0}} R^{3} C_{(k)}\left[\delta_{(k) l} \delta_{m 3}+\delta_{(k) m} \delta_{l 3}\right], \quad C_{1}=C_{2}=\frac{4}{\pi} \frac{1}{2-v_{0}}, \quad C_{3}=0
$$

where brackets around an index mean that no summation over this index is presumed. In the global co-ordinate set $\left(x_{1}, x_{2}, x_{3}\right)$ this tensor can be written as follows

$$
J_{k l m}=J_{p q r}^{\prime} g_{p k} g_{q l} g_{r m}
$$

Here the directional cosines of the crack co-ordinate set with the respect to the global one can, following Salganik (1973), be written as

$$
\left[g_{i k}\right]=\left[\begin{array}{ccc}
-\cos \psi \cos f \cos \theta-\sin \psi \sin f & -\sin \psi \cos f \cos \theta+\cos \psi \sin f & \cos f \sin \theta \\
-\cos \psi \sin f \cos \theta+\sin \psi \cos f & -\sin \psi \sin f \cos \theta+\cos \psi \cos f & \sin f \sin \theta \\
\cos \psi \sin \theta & \sin \psi \sin \theta & \cos \theta
\end{array}\right]
$$

where $\theta \in[0, \pi]$ is the angle between axes $x^{\prime}{ }_{3}$ and $x_{3}, \psi \in[0,2 \pi]$ is the angle between planes $\left(x^{\prime}{ }_{3} x^{\prime}{ }_{1}\right)$ and $\left(x^{\prime}{ }_{3} x_{3}\right), f \in[0,2 \pi]$ is the angle between planes $\left(x_{3} x_{1}\right)$ and $\left(x_{3} x^{\prime}{ }_{3}\right)$.

For the isotropic original material the tensor of compliances can be expressed in the form 
$S_{i k l m}^{0}=\frac{1}{E_{0}}\left\{-v_{0} \delta_{i k} \delta_{l m}+\frac{1}{2}\left(1+v_{0}\right)\left(\delta_{i m} \delta_{l k}+\delta_{i l} \delta_{k m}\right)\right\}$

Substituting equations (A5)-(A8) into equation (A2), taking into account that $g_{3 m}=n_{m}$, that the columns of matrix (A7) are orthogonal and that for isotropic distribution of crack orientations

$$
d Y=\frac{1}{8 \pi^{2}} \sin \theta d \theta d f d \psi d R,
$$

one obtains

$$
S_{i k l m}=\frac{1}{E_{0}}\left\{-\left[v_{0}+\frac{32}{45} \frac{1-v_{0}^{2}}{2-v_{0}} v\right] \delta_{i k} \delta_{l m}+\frac{1}{2}\left[1+v_{0}+\frac{32}{15} \frac{1-v_{0}^{2}}{2-v_{0}} v\right]\left(\delta_{i m} \delta_{l k}+\delta_{i l} \delta_{k m}\right)\right\}
$$

where

$$
v=N \int R^{3} F(R) d R=N\left\langle R^{3}\right\rangle
$$

is the dimensionless crack concentration; $\left\langle R^{3}>\right.$ is the cube of the crack radius averaged over all crack sizes and orientations.

Comparing (A10) and (A9) one finally obtains the expressions for the effective Young's modulus and Poisson's ratio: 


$$
\begin{aligned}
& E=E_{0}\left[1-\frac{64}{45} \frac{1-v_{0}^{2}}{2-v_{0}} v\right] \\
& v=v_{0}+\frac{32}{45} \frac{1-v_{0}^{2}}{2-v_{0}}\left(1-2 v_{0}\right) v
\end{aligned}
$$


Table 1. Summary of self-similar distributions introduced

\begin{tabular}{|l|l|l|}
\hline Distribution & Exponent & Prefactor \\
\hline$f(R)$ - distribution of sliding zones & -3 & $\lambda=3 /(2 \pi)$ \\
\hline$g(R)$ - fraction of sliding zones grown to & $-k$ & $\gamma$ \\
radius $R$ & & \\
\hline$f(R) g(R)$ - distribution of sliding zones & $-(3+k)$ & $\lambda \gamma$ \\
grown to radius $R$ & & \\
\hline E $(R)$ - energy emitted by the sliding zone & $9 / 2$ & $\kappa$ \\
of radius $R$ & & \\
\hline
\end{tabular}




\section{Figure captions}

Fig. 1. Schematic representation of a fault with a sliding zone. A cross section is shown. The sliding zone with cross-sectional dimensions BB' is developed from an initial sliding zone CC'. It is assumed that the large-scale friction in the sliding zone is provided by interacting asperities with negligible friction between the asperity surfaces, such that the sliding over this part of the fault could, in the first approximation be assumed reversible.

Fig. 2. A model of the sliding zone: (a) a representation of the sliding zone as a shear crack loaded at the central part (marked in white) of area $S$ which corresponds to the initial sliding zone; (b) modelling the sliding zone as a shear crack loaded by a pair of concentrated forces of magnitude $F=S\left(\tau-\tau_{\mathrm{I}}\right)$, the angle $\theta$ is a polar angle

Fig. 3. Coplanar sliding zones in a fault: a cross sectional view 